\title{
Photowritten gratings in ion-exchanged glass waveguides
}

\author{
J. E. Roman and K. A. Winick \\ Department of Electrical Engineering and Computer Science, University of Michigan, Ann Arbor, Michigan 48109
}

Received November 16, 1992

\begin{abstract}
The fabrication of an ion-exchanged waveguide beam deflector containing a photowritten grating is described. The planar waveguide was fabricated by thermal $\mathrm{K}^{+}$exchange in a borosilicate glass. The grating was written by photobleaching an absorption defect centered at $330 \mathrm{~nm}$, which was created by $\gamma$-ray irradiation of the glass. The bleaching was accomplished with the 351-nm line from an argon laser. The device achieved 35\% deflection efficiency at $633 \mathrm{~nm}$, which corresponded to a grating with a photoinduced index change of $2.6 \times 10^{-5}$. This is to our knowledge the first demonstration of an ion-exchanged glass waveguide device containing a permanent photowritten grating.
\end{abstract}

Grating-based waveguide devices find many applications, including directional coupling, signal filtering, multiplexing, beam deflection, and distributedfeedback lasing. Ion-exchanged glass waveguide devices are particularly attractive because their fabrication is relatively simple and inexpensive. Recently, an ion-exchanged, single-longitudinal-mode, distributed-Bragg-reflector waveguide laser was reported. ${ }^{1}$ In this laser, the waveguide was fabricated by thermal $\mathrm{Ag}^{+}$-ion diffusion, and the Bragg reflector was argon-ion milled into the glass through a photoresist mask.

Index-modulation gratings are desirable over ionmilled gratings because they can be written directly and noninvasively into the entire waveguide core without the need for photoresist masks. With this type of grating, a refractive-index change of $>10^{-5}$ in the waveguide core should allow fabrication of useful grating-based devices for integrated optics.

Index-modulation gratings can be written in glasses with high-energy particle beams. In this technique, the grating is written line by line with an electron, ion, or neutron beam. The particle bombardment induces a refractive-index change in the glass. ${ }^{2}$ Gratings can also be written optically in photosensitive glasses. In this case, the refractive-index changes are induced by exposing the glass to an optical beam. ${ }^{3}$ This technique is particularly attractive because it allows the use of simple holographic techniques to write high-resolution uniform gratings. In addition, if very high resolution is not required, the technique can be easily used to write aperiodic gratings line by line.

Gratings have been optically written in photosensitive fibers. Line-by-line techniques have been used to write gratings in $\mathrm{Eu}^{2+}$-doped fibers, ${ }^{4}$ and holographic techniques have been used to write gratings in $\mathrm{Ge}$ - and $\mathrm{Ce}^{3+}$-doped fibers. ${ }^{5,6}$ In the case of germanosilica fibers, refractive-index changes as large as $10^{-3}$ have been achieved by photobleaching an absorption band centered at $244 \mathrm{~nm}$, which has been attributed to a $\mathrm{GeO}$ defect center. Photobleaching of this band leads to a refractiveindex change through the Kramers-Kronig relation. With this principle, distributed Bragg-reflector fiber lasers have been reported in $\mathrm{Nd}^{3+}$ - and $\mathrm{Er}^{3+}$ doped germanosilica fibers. ${ }^{7,8}$ More recently, Bragg reflectors have been photowritten in germanosilica planar waveguides fabricated by flame hydrolysis. ${ }^{9}$

Gratings have also been holographically written in $\mathrm{Eu}^{3+}$ - and $\mathrm{Pr}^{3+}$-doped bulk silicate glasses. ${ }^{10,11}$ The permanent index changes reported were of the order of $5 \times 10^{-7}$, which is small for integratedoptical devices. In addition, gratings have been holographically written in bulk silicate glasses by photobleaching radiation-induced absorption centers. ${ }^{11}$ Photoinduced index changes of $<10^{-5}$ were achieved by bleaching a radiation-induced absorption band centered at $450 \mathrm{~nm}$ with the $488-\mathrm{nm}$ line from an argon laser. However, no grating-based waveguide devices were reported.

In this Letter we report on the fabrication of a $\mathrm{K}^{+}$-exchanged glass-waveguide beam deflector with a permanent photowritten grating. This is to our knowledge the first report of an optically written grating in an ion-exchanged glass waveguide on a planar substrate. The grating was fabricated by photobleaching a radiation-induced absorption band centered at $330 \mathrm{~nm}$ with the $351-\mathrm{nm}$ line

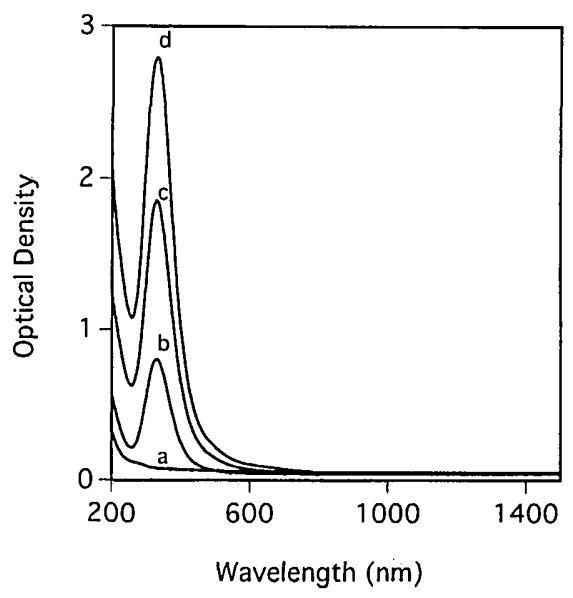

Fig. 1. Absorption spectrum for unirradiated and irradiated 970ANR glass; the sample thickness is $0.8 \mathrm{~mm}$. The doses are $0,1,10$, and $100 \mathrm{Mrad}$ for curves a-d, respectively. 


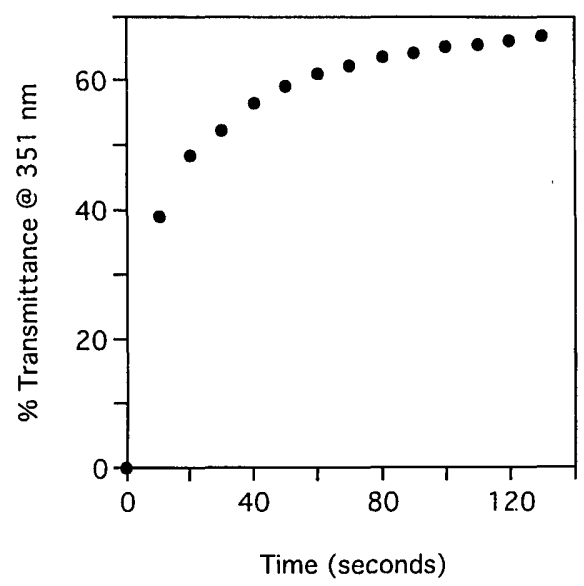

Fig. 2. Photobleaching of a radiation-induced band in 970ANR glass. The sample thickness is $0.8 \mathrm{~mm}$, and the dose is 100 Mrad.

from a small-frame argon laser. The device had a deflection efficiency of $35 \%$ at $633 \mathrm{~nm}$, which corresponded to a photoinduced index change of $2.6 \times 10^{-5}$. This change was achieved with writing intensities of $8 \mathrm{~W} / \mathrm{cm}^{2}$ and exposure times of $30 \mathrm{~min}$. Photobleaching experiments on the irradiated glass and device fabrication are described below.

The glass used was an aluminoborosilicate glass from Corning (experimental melt 970ANR D-8-971). The glass contained sodium, which allowed fabrication of waveguides by ion exchange. The low UV absorption of the glass permitted the study of radiation-induced defects down to $200 \mathrm{~nm}$. Figure 1 shows the absorption spectrum for unirradiated and $\gamma$-ray $\left({ }^{60} \mathrm{Co}\right.$ source)-irradiated samples, as measured in a Cary 2400 spectrometer. The irradiated samples showed an absorption band centered at $330 \mathrm{~nm}$ with a FWHM of $100 \mathrm{~nm}$. The absorption peak increased with the radiation dose, showing no signs of saturation with doses of up to $100 \mathrm{Mrad}$. We found the absorption peak at $330 \mathrm{~nm}$ for the irradiated 970ANR glass to be larger than the absorption peak at $450 \mathrm{~nm}$ for the irradiated silicate glass used in Ref. 11. This suggests that a larger photoinduced index change may be obtained in our glass than has been reported in other silica glasses.

Photobleaching experiments were performed on the irradiated 970ANR samples. Light from an argon laser operating at $351 \mathrm{~nm}$ was focused onto the sample, and the transmitted light was monitored. Figure 2 shows the photobleaching of a $0.8-\mathrm{mm}$ thick sample that was irradiated with a 100-Mrad dose of $\gamma$ rays. The sample was bleached with a $250-\mathrm{mW}$ beam focused to a $10-\mu \mathrm{m}$ waist for an intensity of $300 \mathrm{~kW} / \mathrm{cm}^{2}$. As the figure shows, a 0.4-60\% transmittance change was achieved in just $60 \mathrm{~s}$. This change corresponds to virtually complete bleaching of the 100-Mrad absorption band shown in Fig. 1.

The index change, $\Delta n_{\text {eff }}$, induced by photobleaching can be calculated from the Kramers-Kronig relation given by

$$
\Delta n_{\mathrm{eff}}(\lambda)=\frac{1}{2 \pi^{2}} P \int_{0}^{\infty} \frac{\Delta \alpha_{\mathrm{eff}}\left(\lambda^{\prime}\right)}{1-\left(\lambda^{\prime} / \lambda\right)^{2}} \mathrm{~d} \lambda^{\prime},
$$

where $P$ is the principal part of the integral, $\lambda$ is the wavelength, and $\Delta \alpha_{\text {eff }}(\lambda)$ is the effective change in the absorption coefficient $\left(\mathrm{m}^{-1}\right)$ of the defect, which is given by

$$
\Delta \alpha_{\mathrm{eff}}(\lambda)=(1 / L) \int_{0}^{L} \Delta \alpha(\lambda, z) \mathrm{d} z
$$

where $L$ is the sample thickness. Equation (2) takes into account the fact that the bleaching beam is strongly attenuated as it passes through the sample, and thus bleaching does not occur uniformly in depth. The change in the absorption coefficient can be related to the sample transmittance before and after bleaching by

$$
T_{\mathrm{bl}}(\lambda)=T_{\mathrm{unbl}}(\lambda) \exp \left[\Delta \alpha_{\mathrm{eff}}(\lambda) L\right],
$$

where $T_{\text {unbl }}(\lambda)$ and $T_{\mathrm{bl}}(\lambda)$ are the sample transmittances before and after bleaching, respectively.

Equation (1) was used to calculate the index change induced by completely bleaching the 100-Mrad curve in Fig. 1. In this calculation, $\Delta \alpha_{\text {eff }}(\lambda)$ was modeled as a Gaussian centered at $330 \mathrm{~nm}$ with a FWHM of $100 \mathrm{~nm}$ and a peak value of $8000 \mathrm{~m}^{-1}$. The results suggest that index changes of $6 \times 10^{-5}$ could be induced at $633 \mathrm{~nm}$. Such index changes are large enough to make useful grating devices in waveguides.

The next step was to write gratings holographically in the $\gamma$-ray-irradiated 970ANR samples and to measure the photoinduced index changes. A 2-mmdiameter beam from an 190-5 Coherent argon laser operating at $351 \mathrm{~nm}$ in a single longitudinal mode was split into two beams. The beams were then recombined at the sample, forming a periodic intensity pattern. The angle of incidence was kept small $\left(<3^{\circ}\right)$ to ensure that the incident beams overlapped throughout the sample thickness. The photowritten grating was then probed at $488,514,633,800$, and $1060 \mathrm{~nm}$. For small incidence angles, the diffraction efficiency of the grating at the Bragg angle is given by

$$
\eta=\sin ^{2}\left[\pi L\left(\Delta n_{\mathrm{eff}} / 2\right) / \lambda\right],
$$

where $L$ is the sample thickness, $\lambda$ is the probe wavelength, and $\Delta n_{\text {eff }}$ is the effective photoinduced

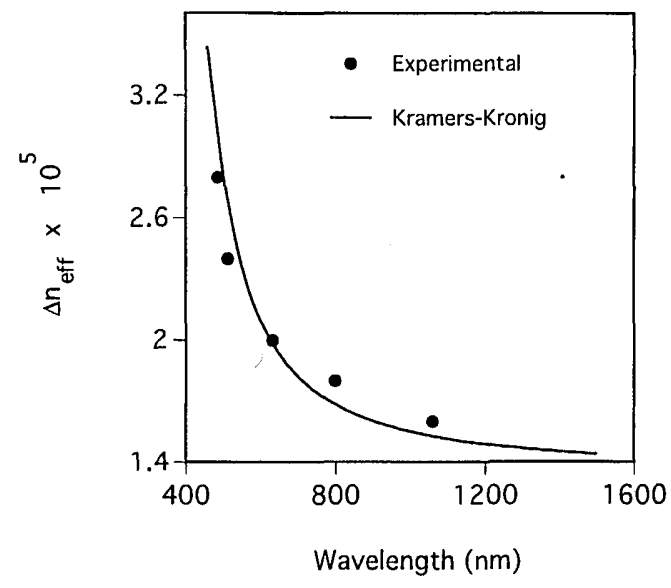

Fig. 3. Photoinduced index change in 970ANR glass. 


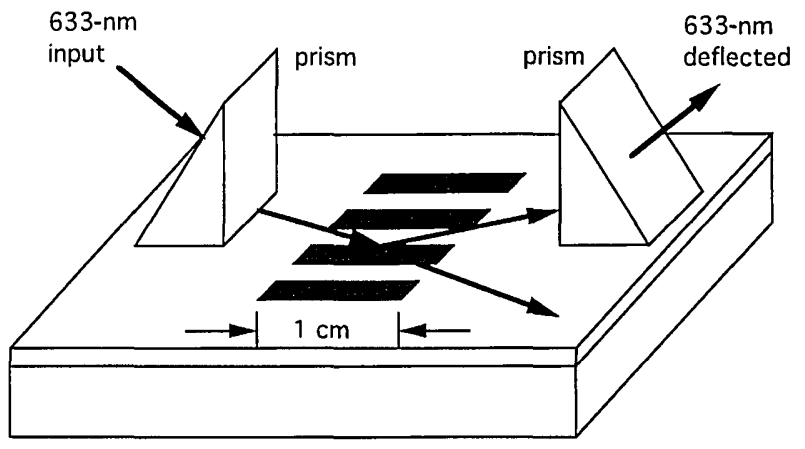

Fig. 4. Waveguide beam deflector.

index change (peak to peak), given by

$$
\Delta n_{\text {eff }}(\lambda)=(1 / L) \int_{0}^{L} \Delta n(\lambda, z) \mathrm{d} z .
$$

The effective photoinduced index change was then calculated by measuring the diffraction efficiency of the grating and using Eq. (4).

Figure 3 shows the measured index changes at several wavelengths for a grating written holographically with an intensity of $0.7 \mathrm{~W} / \mathrm{cm}^{2}$ in each beam and an exposure time of $60 \mathrm{~min}$. The radiation dose for the $130-\mu \mathrm{m}$-thick sample was $100 \mathrm{Mrad}$. The solid curve represents a Kramers-Kronig calculation, where $\Delta \alpha_{\text {eff }}(\lambda)$ was modeled as a Gaussian centered at $330 \mathrm{~nm}$ with a FWHM of $100 \mathrm{~nm}$ and a peak value of $2500 \mathrm{~m}^{-1}$. The peak value was chosen to give the best fit to the experimental data, and it represents a third of the maximum value $\left(8000 \mathrm{~m}^{-1}\right)$. For these parameters, Eq. (3) predicted a 33\% transmittance change at $351 \mathrm{~nm}$, which agreed reasonably well with the $40 \%$ change measured experimentally. These results show that the Kramers-Kronig model correctly predicts the photoinduced index changes and that these changes can be increased by a factor of 3 with higher writing intensities or longer exposure times.

Annealing experiments were also performed on the photowritten gratings. The samples were placed in an oven and annealed for $1 \mathrm{~h}$. After annealing, the diffraction efficiency was remeasured. At $50^{\circ} \mathrm{C}$, no grating erasure was observed. However, a slight decrease in the diffraction efficiency was noted at $100{ }^{\circ} \mathrm{C}$, with complete grating erasure at temperatures of $>150^{\circ} \mathrm{C}$.

The fabrication of the beam deflector proceeded as follows. A single-mode $\mathrm{K}^{+}$-exchanged planar waveguide was fabricated by immersing the substrate in a molten potassium nitrate bath at $380^{\circ} \mathrm{C}$ for $1 \mathrm{~h}$. After the diffusion, a 500-nm-thick aluminum mask with a period of $26.4 \mu \mathrm{m}$ and 1-cm-long lines was evaporated onto the waveguide surface with lift-off lithography. The sample was then irradiated with $100 \mathrm{Mrad}$ of $\gamma$ rays from a ${ }^{60} \mathrm{Co}$ source. Finally, the periodic pattern was transferred to the waveguide by exposing the mask to a $140-\mathrm{mW}$ beam from the UV argon laser operating at $351 \mathrm{~nm}$. The bleaching intensity and exposure time were $8 \mathrm{~W} / \mathrm{cm}^{2}$ and $30 \mathrm{~min}$, respectively. After bleaching, the aluminum mask was removed with aluminum etchant. Figure 4 shows a picture of the device. Light from a $5-\mathrm{mW}$ $\mathrm{He}-\mathrm{Ne}$ laser was prism coupled into the waveguide, and the diffracted light was prism coupled out. The device achieved $35 \%$ deflection efficiency at $633 \mathrm{~nm}$, which corresponded to a photoinduced index change of $2.6 \times 10^{-5}$, with $L=1 \mathrm{~cm}$ in Eq. (4).

In conclusion, we have demonstrated what is to our knowledge the first ion-exchanged waveguide device containing a permanent photowritten grating. The grating was written by photobleaching a radiationinduced absorption band centered at $330 \mathrm{~nm}$ with the 351-nm line from an argon laser. The beam deflector achieved $35 \%$ diffraction efficiency at $633 \mathrm{~nm}$, which corresponded to a photoinduced index change of $2.6 \times 10^{-5}$. This change was achieved with writing intensities of $8 \mathrm{~W} / \mathrm{cm}^{2}$ and writing times of $30 \mathrm{~min}$. Larger index changes and considerably shorter exposure times are possible with higher writing intensities. As a potential application, the 970ANR glass used in this research could be doped with rareearth ions to make a distributed-Bragg-reflector ionexchanged waveguide laser. The grating reflector would be written holographically with the 351-nm line from an argon laser without any need for frequency-doubled sources, as has been required for writing gratings holographically in photosensitive fibers. ${ }^{5,6}$

This research is supported by the National Science Foundation. The authors acknowledge useful discussions with G. M. Williams of the Naval Research Laboratory. The authors also thank Corning for supplying the glass samples and Robert Blackburn from the Phoenix Laboratory at the University of Michigan for irradiating them.

\section{References}

1. J. E. Roman and K. A. Winick, Appl. Phys. Lett. 61, 2744 (1992).

2. D. Barbier, M. Green, and S. Madden, J. Lightwave Technol. 9, 715 (1991).

3. J. Albert, K. O. Hill, B. Malo, D. C. Johnson J. L. Brebner, Y. B. Trudeau, and G. Kajrys, Appl. Phys. Lett. 60, 148 (1992).

4. K. O. Hill, B. Malo, F. Bilodeau, D. C. Johnson, T. F. Morse, A. Kilian, L. Reinhart, and $\mathrm{K}$. Oh, in Optical Fiber Communication, Vol. 4 of 1991 OSA Technical Digest Series (Optical Society of America, Washington, D.C., 1991), paper PD3.

5. G. Meltz, W. W. Morey, and W. H. Glenn, Opt. Lett. 14, 823 (1989).

6. M. M. Broer, R. L. Cone, and J. R. Simpson, Opt. Lett. 16, 1391 (1991).

7. G. A. Ball, W. W. Morey, and J. P. Waters, Electron. Lett. 26, 1829 (1990).

8. G. A. Ball and W. W. Morey, Opt. Lett. 17, 420 (1992).

9. G. D. Maxwell, R. Kashyap, B. J. Ainslie, D. L. Williams, and J. R. Armitage, Electron. Lett. 28, 2106 (1992).

10. M. M. Broer, A. J. Bruce, and W. H. Grodkiewicz, Phys. Rev. B 45, 7077 (1992).

11. G. M. Williams, D. A. Dutt, J. A. Ruller, and E. J. Friebele, Opt. Lett. 17, 532 (1992). 